\title{
Time course of training-induced microcirculatory changes and of VEGF expression in skeletal muscles of spontaneously hypertensive female rats
}

\author{
S.L. Amaral ${ }^{1}$, L.S. Sanchez ${ }^{2}$, A.J.B.A. Chang ${ }^{2}$, L.V. Rossoni² and L.C. Michelini² \\ 1'Departamento de Educação Física, Faculdade de Ciências de Bauru, Universidade Estadual Paulista \\ Júlio de Mesquita Filho, Bauru, SP, Brasil \\ 2Departamento de Fisiologia e Biofísica, Instituto de Ciências Biomédicas, Universidade de São Paulo, \\ São Paulo, SP, Brasil \\ Correspondence to: S.L. Amaral, Departamento de Educação Física, UNESP, Av. Eng. Luiz Edmundo \\ Carrijo Coube, s/n, 17033-360 Bauru, SP, Brasil \\ Fax: +55-14-3103-6082. E-mail: slamaral@fc.unesp.br
}

Exercise-induced vessel changes modulate arterial pressure (AP) in male spontaneously hypertensive rats (SHR). Vascular endothelial growth factor (VEGF) is important for angiogenesis of skeletal muscle. The present study evaluated the time course of VEGF and angiogenesis after short- and long-term exercise training of female SHR and Wistar Kyoto (WKY) rats, 8-9 weeks $(200-250 \mathrm{~g})$. Rats were allocated to daily training or remained sedentary for 3 days $(\mathrm{N}=23)$ or 13 weeks $(\mathrm{N}=23)$. After training, the carotid artery was catheterized for AP measurements. Locomotor (tibialis anterior and gracilis) and non-locomotor skeletal muscles (temporalis) were harvested and prepared for histologic and protein expression analyses. Training increased treadmill performance by all groups (SHR $=28 \%$, WKY $=64 \%, 3$ days) and (SHR $=141 \%$, WKY $=122 \%, 13$ weeks). SHR had higher values of AP than WKY $(174 \pm 4$ vs $111 \pm 2 \mathrm{mmHg})$ that were not altered by training. Three days of running increased VEGF expression (SHR $=28 \%$, WKY $=36 \%$ ) simultaneously with an increase in capillary-to-fiber ratio in gracilis muscle (SHR $=19 \%$, WKY $=15 \%)$. In contrast, 13 weeks of training increased gracilis capillary-to-fiber ratio (SHR $=18 \%$, WKY $=19 \%)$, without simultaneous changes in VEGF expression. Training did not change VEGF expression and capillarity of temporalis muscle. We conclude that training stimulates time- and tissue-dependent VEGF protein expression, independent of pressure levels. VEGF triggers angiogenesis in locomotor skeletal muscle shortly after the exercise starts, but is not involved in the maintenance of capillarity after long-term exercise in female rats.

Key words: High blood pressure; Exercise training; Capillary density; VEGF expression; Skeletal muscle angiogenesis

Research supported by FAPESP (\#03/01578-4 and \#02/05761-5, post-doctoral fellowship to S.L. Amaral) and CNPq (\#304178/ 2005-5 and undergraduate fellowship to L.S. Sanchez).

Received April 16, 2007. Accepted April 30, 2008

\section{Introduction}

Aerobic exercise training promotes many adjustments in skeletal muscle and the cardiovascular system. Some of these include an increase in number and size of mitochondria as well as an increase in oxidative enzyme activities. To preserve the functional balance between metabolic demand and oxygen delivery, the skeletal and cardiac muscles increase capillary density and/or capillary-to-fiber ratio. The increased capillary network plays an important role to improve aerobic capacity, facilitate oxygen transport, conductance, and muscle extraction, therefore contributing to increase maximal oxygen uptake and physical performance (1). It has been shown that microcirculation 
can adapt very rapidly after exercise or electrical stimulation in normotensive male rats (2-6). However, there is very little information regarding whether gender or hypertension may alter this process.

Angiogenesis is a complex process mediated by several metabolic alterations occurring during muscle activity $(7,8)$, increased levels and/or chronic administration of angiotensin II $(2-4,9,10)$, mechanical factors linked to increased shear stress $(8,10)$, wall tension $(8,10)$, hypoxia (11), metalloproteinases (12), reactive oxygen species (13), and several growth factors such as angiopoietin 1 and $2(8,14)$, and the vascular endothelial growth factor (VEGF). VEGF can be stimulated by several factors, including hypoxia $(7,8,11,15,16)$, nitric oxide (17-19), angiotensin II (2-4), and arachidonic acid metabolites (20). Therefore, it has been considered to be an ideal candidate to regulate angiogenesis in physiological and pathological situations (14-16,21,22).

Hypertension is an important risk factor for cardiovascular disease with a major impact on morbidity and mortality (23), characterized by several skeletal muscle abnormalities including decreased vessel density, which could contribute to the reduced aerobic capacity observed in arterial hypertension $(24,25)$. Hypertension-induced alterations on skeletal muscle VEGF expression are not well understood. Results from our laboratory have supported the hypothesis that chronic regular aerobic exercise promotes angiogenesis and structural changes in locomotor muscles, contributing to improve physical capacity in normotensive and hypertensive male animals $(24,25)$.

There is no information regarding exercise adjustments in female hypertensives. Since VEGF is an important fast regulator of angiogenesis, we hypothesized that VEGF, by triggering angiogenesis in the skeletal muscle, could be one of the factors responsible for training-induced capillary growth in female rats. In this paper, we report the pattern of VEGF expression and microcirculatory responses in locomotor and non-locomotor skeletal muscles, which present different metabolic rates and blood flow characteristics during exercise.

\section{Material and Methods}

\section{Animal surgery and experimental protocol}

The Institutional Animal Care and Use Committee of the Biomedical Science Institute, University of São Paulo, approved all surgical procedures and protocols used. The animals were housed at the Animal Facilities of the Biomedical Science Institute and were given water and food ad libitum. Forty-six female Wistar Kyoto (WKY) and spontaneously hypertensive rats (SHR) aged 2 months (200-
$250 \mathrm{~g}$ ) were familiarized to treadmill running for 5 days (5$10 \mathrm{~min} /$ day) before the beginning of training and then randomly assigned to 3 days (group I, $\mathrm{N}=23$ ) or 13 weeks (group II, $\mathrm{N}=23$ ) of training protocols $(\mathrm{T})$ or kept sedentary (S). At the beginning of the protocols, all animals performed maximal tests (26) and T groups were assigned to run on the treadmill at $50-60 \%$ of maximal capacity, $1 \mathrm{~h} /$ day for 3 days or 13 weeks. Maximal tests were repeated after 3 days (group I), and after 6 and 13 weeks (group II) in order to re-adapt the velocity, thus maintaining the intensity of training and to compare the efficacy of $T$ vs $S$ protocols. Body weight and tail pressure were measured throughout the study.

At the end of protocols, rats were anesthetized with a mixture of ketamine, xylazine and acepromazine (0.7:0.2: $0.1, \mathrm{v}: \mathrm{v}, 0.04 \mathrm{~mL} / \mathrm{kg}, \mathrm{im})$. Animals were euthanized by an overdose of anesthesia. Gracilis and tibialis anterior muscles (locomotor) and temporalis muscle (non-locomotor) were quickly removed. Half of each tissue was immediately frozen in dry ice and the other half fixed (4\% paraformaldehyde immersion) for protein expression and histological procedures.

\section{Morphological analysis of vessel density}

Fixed tissues were dehydrated with ethanol and xylol, cut into 5- $\mu \mathrm{m}$ thick sections and stained with hematoxylin and eosin for morphometric analysis (25). Transverse sections of skeletal muscle were analyzed with a light microscope (200X magnification, H 500, Helmut Hund, Wetzlar, Germany). Occurrence of capillaries (up to 12 $\mu \mathrm{m}$ ) was analyzed on digitalized images (Sony CCD IRIS/ RGB color video camera model DXC-151A, Sony Electronics Inc., USA) from 10-15 microscopic fields/tissue randomly chosen and quantified off line (Media Cybernetics, Pro-Series 128 Capture Kit software, Image Plus, USA) $(24,25)$. Capillary density was reported as capillaryto-fiber ratio.

\section{Western blot analysis to detect the presence of VEGF protein}

Tibialis anterior and temporalis muscles $(100 \mathrm{mg})$ were homogenized and protein suspended in $0.1 \mathrm{M}$ potassium buffer $\left(\mathrm{KPO}_{4}\right)$ for the Western blot procedure (3). In summary, $80 \mu \mathrm{g}$ protein from each tissue and from kidney (used as positive control) were separated on a $12 \%$ denaturing polyacrylamide gel. The gels were transferred to a nitrocellulose membrane and blocked with $5 \%$ non-fat dry milk (3). After blocking, blots were incubated with a monoclonal antibody to a peptide derived from the human VEGF sequence (1:1,000 dilution, clone G143-850, Pharmingen, BD Biosciences, USA) overnight at $4^{\circ} \mathrm{C}$. Washed blots 
were incubated with goat anti-mouse secondary antibody at a dilution of 1:10,000 (1 h) at room temperature and then submitted to a luminal substrate (Santa Cruz Biotechnology, USA) detection system. Membranes were exposed to X-ray film (Fuji Medical, USA) for 2-3 min and developed. The VEGF band intensity was quantified using a Scion Image Beta 4.02 for Windows (Scion Corporation, USA), and values were reported as a percent of the positive control.

\section{Statistical analysis}

All values are reported as means \pm SEM. For each muscle, vessel counts of all the selected fields were averaged to a single vessel density/rat. Vessel density and VEGF protein expression were evaluated using a two-way ANOVA, with the Tukey as a post hoc test. For treadmill performance through the experimental protocol, repeated measures ANOVA was used. Differences were consid- ered to be significant at $\mathrm{P}<0.05$.

\section{Results}

The SHR group had higher arterial pressure from the 1st week $(111 \pm 2,112 \pm 2,174 \pm 4,166 \pm 2 \mathrm{mmHg}$, for WKYS, WKYT, SHRS, and SHRT, respectively; P < 0.05), with no changes during the 3-day period. Training for 13 weeks also did not change the arterial pressure of any group $(118 \pm 1,110 \pm 1,175 \pm 2,168 \pm 2 \mathrm{mmHg}$, for WKYS, WKYT, SHRS, and SHRT, respectively; $P<0.05$ ).

Body weight of the 3-day groups did not change, but significantly increased after 13 weeks (data not shown). As shown in Figure 1, both 3 days and 13 weeks of training at $60 \%$ of physical capacity increased running distance on a treadmill $(\mathrm{WKY}=+64$ and $+121 \%$, SHR $=+28$ and $+141 \%$, for 3 and 13 weeks, respectively; $P<0.05)$.

Western blot analysis showed that VEGF levels were

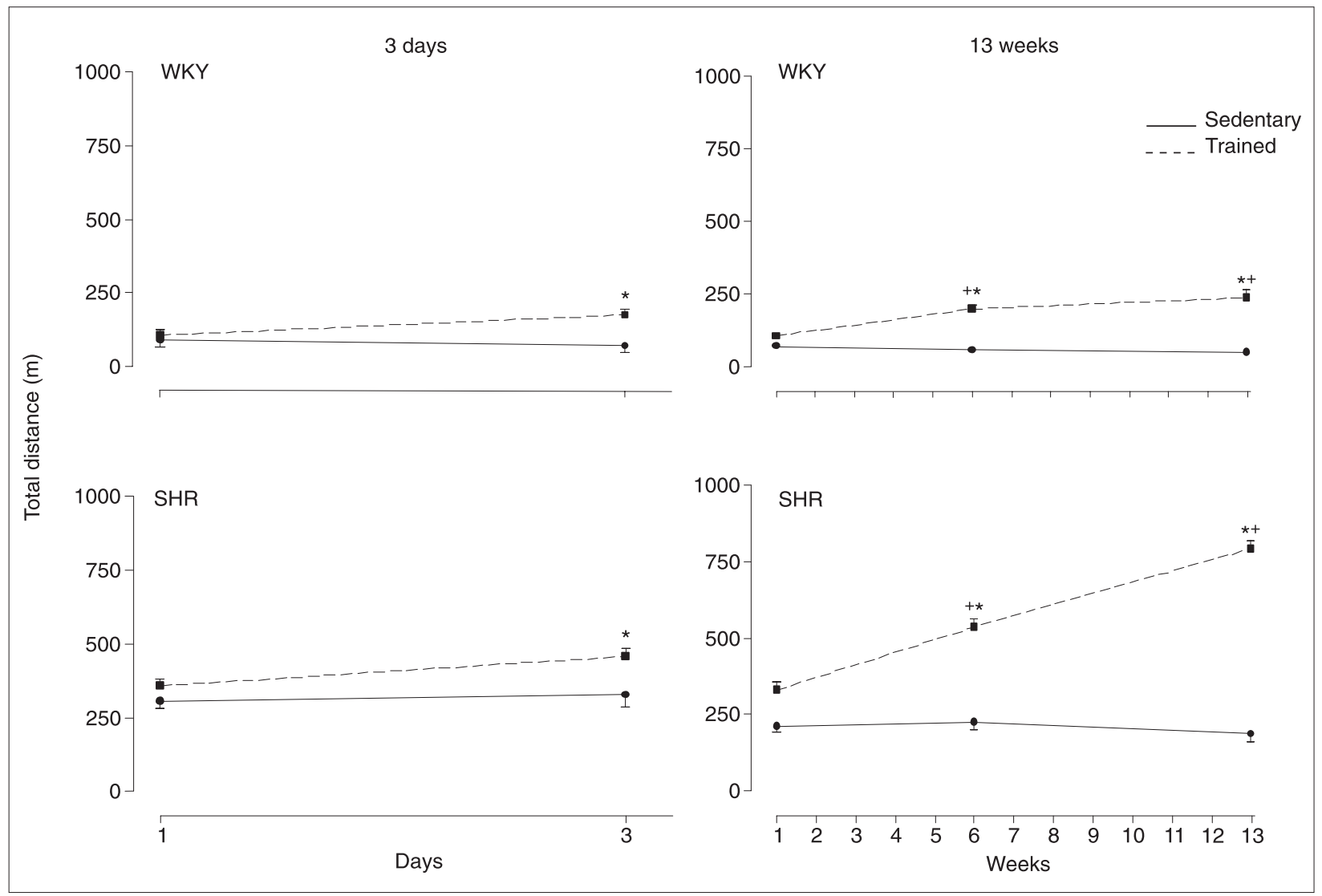

Figure 1. Total distance $(\mathrm{m})$ on treadmill during the training protocol. Left panels, 3 days $(\mathrm{N}=23)$. Right panel, 13 weeks $(\mathrm{N}=23)$. In the 3-day protocol, the maximal test was done at the beginning and at the end of the protocol. In the 13-week protocol, the maximal test was performed at week 0 and repeated at the 6th and 13th weeks. Data are reported as means \pm SEM for 46 rats. WKY $=$ Wistar Kyoto rats $(N=22)$; SHR $=$ spontaneously hypertensive rats $(N=24) .{ }^{*} P<0.05$ compared to sedentary rats at the same time; ${ }^{+} \mathrm{P}<$ 0.05 compared to week 0 (repeated measures one-way ANOVA). 
not different between sedentary WKY and SHR (Figure 2). At the top of each panel there is an illustrative VEGF gel of one rat, which is representative of each group. The densitometry results (average of 6-7 animals) are presented at the bottom of each panel. Training for 3 days increased VEGF expression in locomotor skeletal muscle of both WKY and SHR (36 and 28\%, respectively; $\mathrm{P}<0.05$ ), but not in non-locomotor skeletal muscle. Interestingly, the quick training-induced increase in VEGF expression was not maintained after 13 weeks of training in either of the muscles analyzed, as shown in Figure 2 for locomotor and non-locomotor muscles.

Figure 3 shows digitized images of microvessels from locomotor and non-locomotor muscles taken from representative rats of the 8 experimental groups. Three days or 13 weeks of running increased capillaries only in locomo- tor muscles of both WKY and SHR. Figure 4 presents the quantitative data. Female SHR presented values of capillary-to-fiber ratios and VEGF expression not different from WKY in all muscles analyzed (Figure 2). Three days and 13 weeks of exercise on a treadmill significantly increased vessel density in locomotor muscle of both WKY (15 and $19 \%$ ) and SHR (19 and 18\%). This response, however, was not observed in the non-locomotor muscle (Figure 4).

\section{Discussion}

The main findings of this study are: 1) short-term exercise training increased VEGF protein expression and caused angiogenesis in both normotensive and hypertensive female rats, but only in locomotor skeletal muscles; 2) training-induced VEGF increase was not observed after

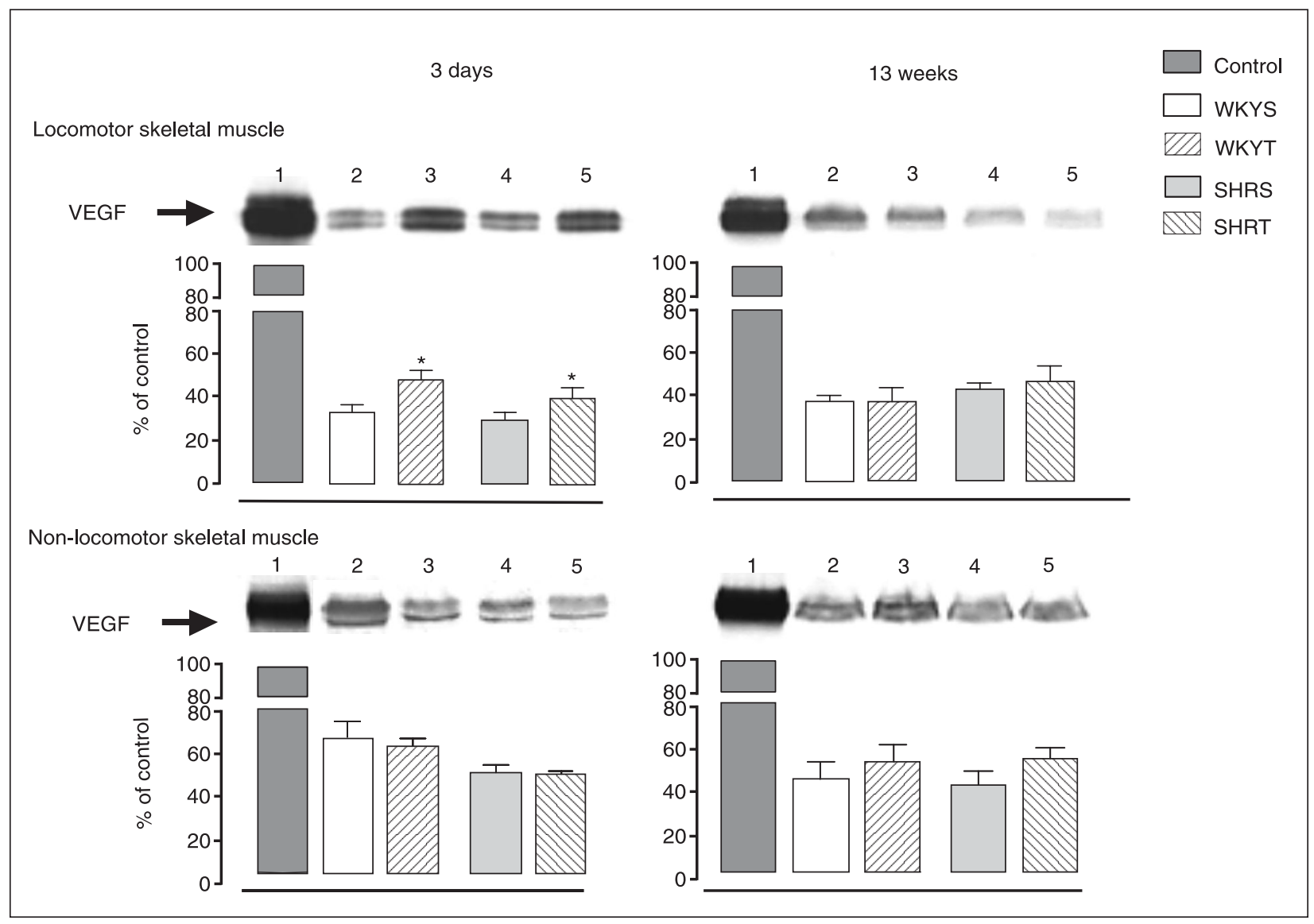

Figure 2. Vascular endothelial growth factor (VEGF) in locomotor and non-locomotor muscles after 3 days (left panels) and 13 weeks (right panels) of exercise. Top of each panel, Total protein $(80 \mu \mathrm{g})$ from a single rat was loaded (illustrative). Lanes of each gel are as follows: lane 1: positive control (mouse kidney); lane 2: WKYS; lane 3: WKYT; lane 4: SHRS; lane 5: SHRT. Bottom of each panel, Quantitative densitometry of VEGF protein in pools of 6-7 rats/group after 3 days (left) or 13 weeks (right) in locomotor (upper panels) and non-locomotor (lower panels) muscles. Data are reported as means \pm SEM for 6-7 rats in each group. WKY = Wistar Kyoto rats; $\mathrm{SHR}=$ spontaneously hypertensive rats; $\mathrm{S}=$ sedentary rats; $\mathrm{T}=$ trained rats. ${ }^{*} \mathrm{P}<0.05$ compared to sedentary rats (two-way ANOVA). 
Locomotor skeletal muscle - 3 days

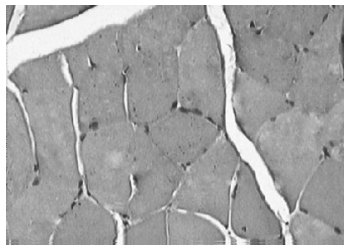

Non-locomotor skeletal muscle - 3 days
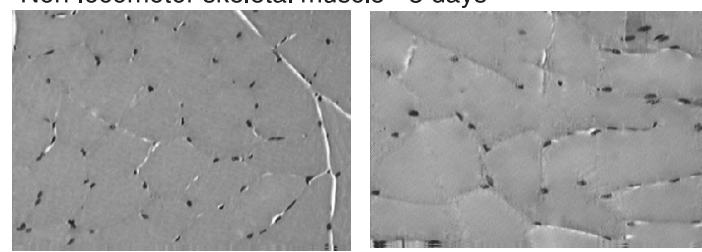

Locomotor skeletal muscle - 13 weeks
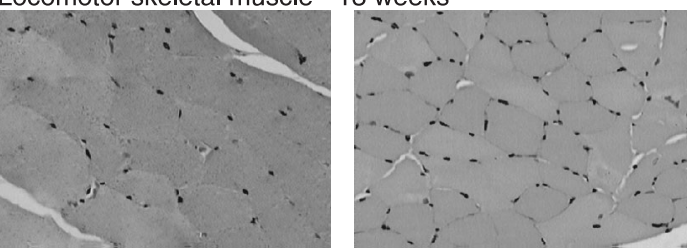

Non-locomotor skeletal muscle - 13 weeks

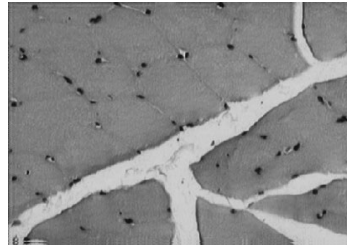

WKYS

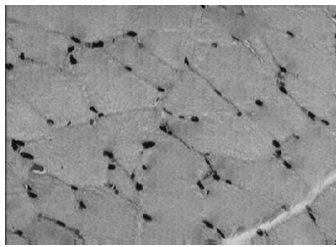

WKYT
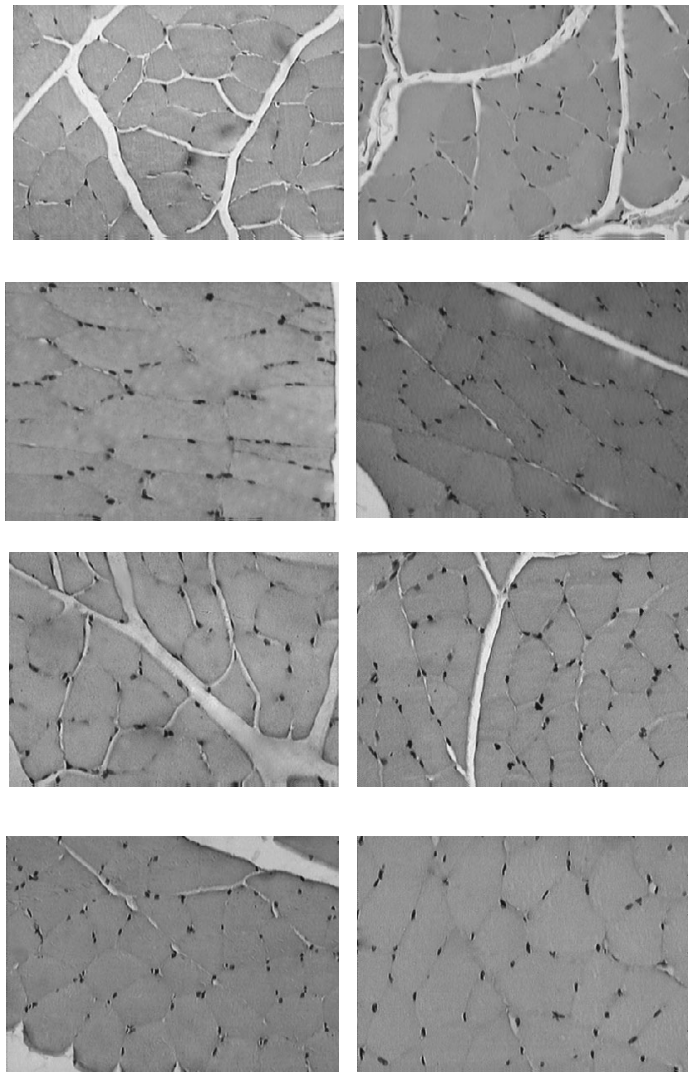

SHRS

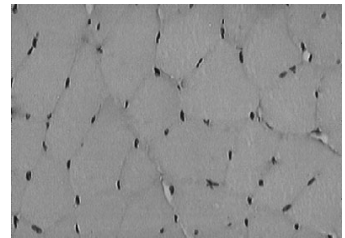

SHRT

Figure 3. Eosin-hematoxylin-stained muscle. The vessels appear black against gray skeletal muscle background. The figure shows illustrative digitized images of microvessels from locomotor and non-locomotor muscles taken from sedentary rats or rats trained for 3 days and 13 weeks. Note the higher number of vessels in both trained groups, WKY and SHR, compared to sedentary ones, in locomotor muscles. In contrast, there is no vessel density changes in non-locomotor muscles. WKY = Wistar Kyoto rats; SHR = spontaneously hypertensive rats; $\mathrm{S}=$ sedentary rats; $\mathrm{T}=$ trained rats.

3 days

Locomotor skeletal muscle

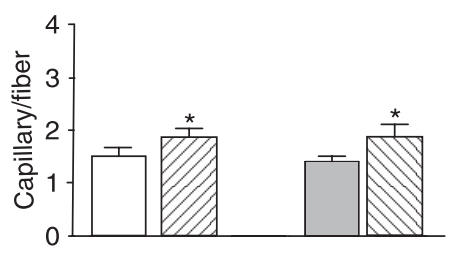

Non-locomotor skeletal muscle
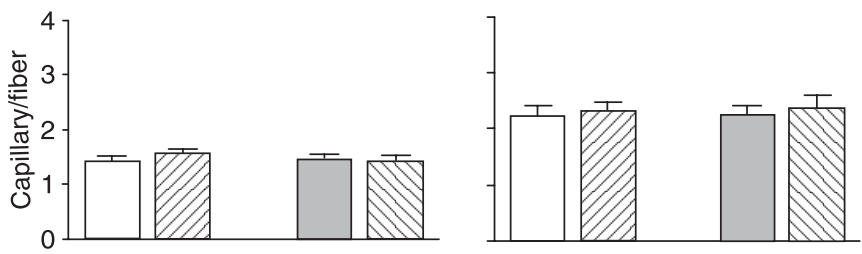

Figure 4. Capillary-to-fiber ratio of rats in locomotor and non-locomotor muscles after 3 days or 13 weeks of exercise. Data are reported as means \pm SEM for 5-6 rats in each group. WKY = Wistar Kyoto rats; SHR = spontaneously hypertensive rats; $\mathrm{S}=$ sedentary rats; $T=$ trained rats. ${ }^{*} \mathrm{P}<0.05 \mathrm{com}$ pared to sedentary rats (two-way ANOVA). 
chronic exercise although the angiogenic response persisted.

Increased capillary network is an important local adjustment to facilitate tissue perfusion (8). Angiogenesis, by increasing capillary exchange area, contributes to increased blood flow and increased oxygen uptake $\left(\mathrm{O}_{2}\right.$ transport, conductance and extraction), thus representing an essential adaptive response of skeletal muscle to exercise for improvement of physical performance. Recently, Richardson et al. (1) demonstrated a good positive correlation between quadriceps maximal $\mathrm{O}_{2}$ extraction, maximal $\mathrm{O}_{2}$ conductance and uptake. The increased capillary density was necessary to compensate for the prolonged metabolic imbalance between metabolic requirement and tissue perfusion during exercise $(1,8)$. Exercise-induced angiogenesis is a well-described response observed in several exercised muscles such as tibialis anterior (2), soleus $(25,27)$, gastrocnemius $(2,25,27)$, vastus lateralis (1), and cardiac muscle $(24,25)$ in male normotensive $(1,2,24,25)$ as well as hypertensive subjects $(24,25)$. A report by our group was the first to demonstrate that non-locomotor muscles, such as the temporalis, did not respond to exercise as the other skeletal muscles (25) in both normotensive and hypertensive male rats. This supports the idea of a local angiogenic response, conditioned by exercise. L-arginine supplementation causes additional effects such as increases in VEGF expression and in capillary-to-fiber ratio on exercise-induced muscles of male rats (28). In the present study, we extended our previous findings by showing that locomotor muscles (not the temporalis) of normotensive and hypertensive female rats also responded to exercise with angiogenesis and increased VEGF expression. In addition, we showed that exercise-induced VEGF expression was a transient response while increased capillary bed was maintained chronically in females, a pattern similar to that observed in male rats.

The process of physiological angiogenesis induced by exercise is controlled by a number of mechanisms (14). According to previous studies, factors like shear stress $(8,10)$, wall tension $(8,10)$, nitric oxide $(14,17-19,28)$, fibroblast growth factor (8), angiotensin II (2-4,9), 20-HETE (18), placental growth factor (8), hypoxia $(7,8,10,11,15,16)$, angiopoietin 1 (27), membrane metalloproteinases (12), and VEGF $(2-4,7,8,10,12,15,17,18,21)$ are effective mechanisms to trigger angiogenesis. Among these factors, VEGF, a 45-kDa homodimeric glycoprotein mitogen, has been considered to be an important regulator of angiogenesis in animals and humans $(2-4,7,8,10,12,15,16,19)$ because it increases vascular permeability, promotes endothelial cell proliferation and stimulates the in vivo formation of new blood vessels $(8,15,16)$. Several investigators have shown that VEGF mRNA and protein are involved in angiogenesis induced after 7 days of electrical stimulation and 3 days of short-term exercise in normotensive male rats (2$4,11,12,18,29)$ and humans $(1,30-32)$, as observed in locomotor muscles such as tibialis anterior, extensor digitorum longus, gastrocnemius, vastus lateralis $(1,6,30-32)$, and cardiac muscle (21). Indeed, we also showed that the angiogenesis induced by electrical stimulation and exercise is dependent on VEGF availability (2-4). The action of VEGF was mainly mediated by two receptors: VEGF-R1 (FIt-1) and VEGF-R2 (Flk-1 or KDR). Although Flt-1 and Flk-1 receptors bind VEGF with high affinity, Flk-1 plays a more important role in VEGF-mediated endothelial cell proliferation $(14,27)$. Most of these studies, however, were conducted in males or men.

In the present study, we extended the observation to female WKY and SHR by showing that increased VEGF expression was present only in locomotor muscles exhibiting higher capillarity following exercise. Our results showed that increased VEGF expression after short term, but not chronic exercise, suggested that an early response to exercise triggers capillary formation thus increasing blood flow, $\mathrm{O}_{2}$ conductance and $\mathrm{O}_{2}$ extraction. In the presence of an already increased capillary formation, the expression of VEGF returned to control levels. This observation confirms the presence of a negative feedback mechanism for VEGF response in female WKY and SHR rats, as proposed by Richardson et al. (1) in humans. Indeed, Annex et al. (33) have suggested that the expression of both mRNA and VEGF protein induced by electrical stimulation is transient, decreasing with time. There are few studies investigating the role of VEGF and VEGF receptors after exercise training even in males. lemitsu et al. (34) reported that training could revert the effects of aging on VEGF and VEGF receptor expression. In a study on time course changes of VEGF, VEGF receptors, and capillary density in male rats submitted to femoral artery ligation and exercise, Lloyd et al. (27) demonstrated the following changes: increased VEGF after 1,3 and 8 days, increased Flk- 1 and Flt- 1 after 3-8 days (which was accompanied by similar changes in endothelial nitric oxide synthase mRNA expression) and increased capillary density only after 12 days of ischemic exercise, when both peptide and receptors were back to control levels. They suggested that exercise training stimulates high transient expression of both VEGF (early response) and its receptors (next step) to trigger capillary angiogenesis, which was maintained during the exercise protocol. Unfortunately, we did not measure receptor expression in our experiments, but we did show that increased VEGF protein expression in female WKY and SHR was accompanied by angiogenesis after 3 days of 
exercise, but only increased capillary density after 3 months. On the basis of these results and the study by Lloyd et al. (27), it is likely that in female WKY and SHR: 1) VEGF receptor expression was increased shortly after or simultaneously with the peptide during the initial phase of exercise, when gene expression changes are large (27); 2) during the chronic phase, the coexistence of both increased capillary bed and reduced gene activation could attenuate the protein expression of VEGF, Flt-1 and Flk-1. Therefore, our results support the proposition of Richardson et al. (1) of a negative feedback mechanism to reduce VEGF (and VEGF receptors) expression in the presence of increased capillary bed after 3 months of training. Another possible explanation for the late decrease of VEGF is related to cellular adaptive responses associated with exercise training. It has been shown that exercise-induced VEGF expression is mediated by at least two other interdependent mechanisms: direct stimulation by hypoxia-in-

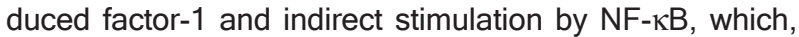
by changing the expression of the inducible form of cyclooxygenase-2 and prostaglandin production, increases VEGF levels (35). Experimental evidence showing that chronic exercise decreases NF- $\mathrm{KB}$ (36) and increases superoxide dismutase, but does not change hypoxia-induced factor-1 (37), also suggested a decreased stimulation for VEGF expression after training.

Our results in female WKY and SHR also suggest that the training-induced VEGF response is independent of pressure changes. Rarefaction or abnormal angiogenesis is normally demonstrated in high-risk hypertensive patients or animals (38), but there are few studies evaluating the levels of VEGF and its correlation with rarefaction/ angiogenesis in hypertension. By using VEGF plasma levels as an index of angiogenesis (without measuring capillary density), Felmeden et al. (38) have demonstrated

\section{References}

1. Richardson RS, Wagner H, Mudaliar SR, Saucedo E, Henry $\mathrm{R}$, Wagner PD. Exercise adaptation attenuates VEGF gene expression in human skeletal muscle. Am J Physiol Heart Circ Physiol 2000; 279: H772-H778.

2. Amaral SL, Papanek PE, Greene AS. Angiotensin II and VEGF are involved in angiogenesis induced by short-term exercise training. Am J Physiol Heart Circ Physiol 2001; 281: $\mathrm{H} 1163-\mathrm{H} 1169$.

3. Amaral SL, Linderman JR, Morse MM, Greene AS. Angiogenesis induced by electrical stimulation is mediated by angiotensin II and VEGF. Microcirculation 2001; 8: 57-67.

4. Amaral SL, Roman RJ, Greene AS. Renin gene transfer restores angiogenesis and vascular endothelial growth factor expression in Dahl S rats. Hypertension 2001; 37: 386- that VEGF plasma levels were increased while VEGF receptor expression was decreased in hypertensive patients compared to normotensive ones. The role of VEGF on both maintenance of capillary density and control of training-induced angiogenesis in hypertensive subjects has not been explored sufficiently. Our results showed neither rarefaction nor decreased VEGF levels in skeletal muscles of female SHR. It is possible that estrogen, by maintaining high VEGF levels (39), could contribute to the absence of rarefaction in female hypertensive rats.

We demonstrated that training stimulates VEGF protein expression in a time- and tissue-dependent manner, independent of pressure levels. Indeed, the data suggest that training stimulates an early muscle recruitment-dependent VEGF expression soon after exercise starts to trigger angiogenesis in locomotor muscles, which is no longer present after long-term exercise when an increased capillary bed is sufficient to maintain blood flow in female exercised rats. These results, revealing the time-course of VEGF angiogenic effects, are of great importance because VEGF therapy has been indicated as treatment for several cardiovascular diseases (40).

\section{Acknowledgments}

The authors thank Sidney Veríssimo Filho (Department of Pharmacology, Biomedical Science Institute, University of São Paulo, São Paulo, SP, Brazil) for expert technical assistance and Dr. Carla Roberta Oliveira Carvalho (Department of Physiology and Biophysics, Biomedical Science Institute, University of São Paulo) and Dr. Jose Roberto Bosqueiro (Department of Physical Education, UNESP, São Paulo State University, Bauru, SP, Brazil) for allowing the use of their laboratories for some experiments.
390.

5. Linderman JR, Kloehn MR, Greene AS. Development of an implantable muscle stimulator: measurement of stimulated angiogenesis and poststimulus vessel regression. Microcirculation 2000; 7: 119-128.

6. Suzuki J. Time-course changes in VEGF expression and capillarity in the early stage of exercise training with Co treatment in rat skeletal muscles. Acta Physiol Scand 2004; 181: 225-232.

7. Risau W. Mechanisms of angiogenesis. Nature 1997; 386: 671-674

8. Prior BM, Yang HT, Terjung RL. What makes vessels grow with exercise training? J Appl Physiol 2004; 97: 1119-1128.

9. Munzenmaier DH, Greene AS. Opposing actions of angio- 
tensin II on microvascular growth and arterial blood pressure. Hypertension 1996; 27: 760-765.

10. Hudlicka O, Brown MD. Postnatal growth of the heart and its blood vessels. J Vasc Res 1996; 33: 266-287.

11. Olfert IM, Breen EC, Mathieu-Costello O, Wagner PD. Skeletal muscle capillarity and angiogenic mRNA levels after exercise training in normoxia and chronic hypoxia. $J$ Appl Physiol 2001; 91: 1176-1184.

12. Brown MD, Hudlicka O. Modulation of physiological angiogenesis in skeletal muscle by mechanical forces: involvement of VEGF and metalloproteinases. Angiogenesis 2003; 6: $1-14$

13. Ushio-Fukai M, Alexander RW. Reactive oxygen species as mediators of angiogenesis signaling: role of $\mathrm{NAD}(\mathrm{P}) \mathrm{H}$ oxidase. Mol Cell Biochem 2004; 264: 85-97.

14. Bloor CM. Angiogenesis during exercise and training. Angiogenesis 2005; 8: 263-271.

15. Ferrara N, Gerber HP, LeCouter J. The biology of VEGF and its receptors. Nat Med 2003; 9: 669-676.

16. Hoeben A, Landuyt B, Highley MS, Wildiers H, Van Oosterom AT, De Bruijn EA. Vascular endothelial growth factor and angiogenesis. Pharmacol Rev 2004; 56: 549-580.

17. Milkiewicz M, Hudlicka O, Brown MD, Silgram H. Nitric oxide, VEGF, and VEGFR-2: interactions in activity-induced angiogenesis in rat skeletal muscle. Am J Physiol Heart Circ Physiol 2005; 289: H336-H343.

18. Husain K. Physical conditioning modulates rat cardiac vascular endothelial growth factor gene expression in nitric oxide-deficient hypertension. Biochem Biophys Res Commun 2004; 320: 1169-1174.

19. Gavin TP, Spector DA, Wagner H, Breen EC, Wagner PD. Nitric oxide synthase inhibition attenuates the skeletal muscle VEGF mRNA response to exercise. J Appl Physiol 2000; 88: 1192-1198.

20. Amaral SL, Maier KG, Schippers DN, Roman RJ, Greene AS. CYP4A metabolites of arachidonic acid and VEGF are mediators of skeletal muscle angiogenesis. Am J Physiol Heart Circ Physiol 2003; 284: H1528-H1535.

21. Jensen L, Pilegaard $H$, Neufer PD, Hellsten Y. Effect of acute exercise and exercise training on VEGF splice variants in human skeletal muscle. Am J Physiol Regul Integr Comp Physiol 2004; 287: R397-R402.

22. Gustafsson $\mathrm{T}$, Ameln H, Fischer $\mathrm{H}$, Sundberg CJ, Timmons JA, Jansson E. VEGF-A splice variants and related receptor expression in human skeletal muscle following submaximal exercise. J Appl Physiol 2005; 98: 2137-2146.

23. Chobanian AV, Bakris GL, Black HR, Cushman WC, Green LA, Izzo JL Jr, et al. The Seventh Report of the Joint National Committee on Prevention, Detection, Evaluation, and Treatment of High Blood Pressure: the JNC 7 report. JAMA 2003; 289: 2560-2572.

24. Amaral SL, Zorn TM, Michelini LC. Exercise training normalizes wall-to-lumen ratio of the gracilis muscle arterioles and reduces pressure in spontaneously hypertensive rats. $J$ Hypertens 2000; 18: 1563-1572.

25. Melo RM, Martinho E Jr, Michelini LC. Training-induced, pressure-lowering effect in SHR: wide effects on circulatory profile of exercised and nonexercised muscles. Hypertension 2003; 42: 851-857.

26. Silva GJ, Brum PC, Negrao CE, Krieger EM. Acute and chronic effects of exercise on baroreflexes in spontane- ously hypertensive rats. Hypertension 1997; 30: 714-719.

27. Lloyd PG, Prior BM, Yang HT, Terjung RL. Angiogenic growth factor expression in rat skeletal muscle in response to exercise training. Am J Physiol Heart Circ Physiol 2003; 284: $\mathrm{H} 1668-\mathrm{H} 1678$.

28. Suzuki J. L-arginine supplementation causes additional effects on exercise-induced angiogenesis and VEGF expression in the heart and hind-leg muscles of middle-aged rats. $J$ Physiol Sci 2006; 56: 39-44

29. Brutsaert TD, Gavin TP, Fu Z, Breen EC, Tang K, MathieuCostello $\mathrm{O}$, et al. Regional differences in expression of VEGF mRNA in rat gastrocnemius following $1 \mathrm{hr}$ exercise or electrical stimulation. BMC Physiol 2002; 2: 8.

30. Gustafsson T, Puntschart A, Kaijser L, Jansson E, Sundberg CJ. Exercise-induced expression of angiogenesis-related transcription and growth factors in human skeletal muscle. Am J Physiol 1999; 276: H679-H685.

31. Gustafsson T, Knutsson A, Puntschart A, Kaijser L, Nordqvist AC, Sundberg CJ, et al. Increased expression of vascular endothelial growth factor in human skeletal muscle in response to short-term one-legged exercise training. Pflugers Arch 2002; 444: 752-759.

32. Wittwer M, Billeter R, Hoppeler H, Fluck M. Regulatory gene expression in skeletal muscle of highly endurance-trained humans. Acta Physiol Scand 2004; 180: 217-227.

33. Annex BH, Torgan CE, Lin P, Taylor DA, Thompson MA, Peters KG, et al. Induction and maintenance of increased VEGF protein by chronic motor nerve stimulation in skeletal muscle. Am J Physiol 1998; 274: H860-H867.

34. lemitsu M, Maeda S, Jesmin S, Otsuki T, Miyauchi T. Exercise training improves aging-induced downregulation of VEGF angiogenic signaling cascade in hearts. Am J Physiol Heart Circ Physiol 2006; 291: H1290-H1298.

35. Lukiw WJ, Ottlecz A, Lambrou G, Grueninger M, Finley J, Thompson HW, et al. Coordinate activation of HIF-1 and NF-kappaB DNA binding and COX-2 and VEGF expression in retinal cells by hypoxia. Invest Ophthalmol Vis Sci 2003; 44: 4163-4170.

36. Sriwijitkamol A, Christ-Roberts C, Berria R, Eagan P, Pratipanawatr T, DeFronzo RA, et al. Reduced skeletal muscle inhibitor of kappaB beta content is associated with insulin resistance in subjects with type 2 diabetes: reversal by exercise training. Diabetes 2006; 55: 760-767.

37. Ookawara T, Suzuk K, Haga S, Ha S, Chung KS, Toshinai $\mathrm{K}$, et al. Transcription regulation of gene expression in human skeletal muscle in response to endurance training. Res Commun Mol Pathol Pharmacol 2002; 111: 41-54.

38. Felmeden DC, Spencer CG, Blann AD, Beevers DG, Lip GY. Physical activity in relation to indices of endothelial function and angiogenesis factors in hypertension: a substudy of the Anglo-Scandinavian Cardiac Outcomes Trial (ASCOT). J Intern Med 2003; 253: 81-91.

39. Bausero P, Ben-Mahdi M, Mazucatelli J, Bloy C, PerrotApplanat M. Vascular endothelial growth factor is modulated in vascular muscle cells by estradiol, tamoxifen, and hypoxia. Am J Physiol Heart Circ Physiol 2000; 279: H2033$\mathrm{H} 2042$.

40. Yla-Herttuala S, Rissanen TT, Vajanto I, Hartikainen J. Vascular endothelial growth factors: biology and current status of clinical applications in cardiovascular medicine. $J$ Am Coll Cardiol 2007; 49: 1015-1026. 